\title{
Manipulation of Working Memory Information Is Impaired in Parkinson's Disease and Related to Working Memory Capacity
}

\author{
P. Bublak and U. Müller \\ Max Planck Institute of Cognitive Neuroscience \\ M. Reuter \\ Neurological Hospital Martha-Maria Halle/Saale
}

\author{
G. Grön \\ University of Leipzig \\ D. Y. von Cramon \\ Max Planck Institute of Cognitive Neuroscience and \\ University of Leipzig
}

\begin{abstract}
It has been suggested that in patients with Parkinson's disease (PD), difficulties in the manipulation of information, which result in problems in executive tasks, are related to a reduction of working memory capacity (J. D. E. Gabrieli, J. Singh, G. T. Stebbins, \& C. G. Goetz, 1996). The present study selectively varied the manipulation demand irrespective of the maintenance requirement. In a group of 14 PD patients, performance declined overproportionally with the increasing task demand and was significantly correlated with a measure of working memory capacity. These results suggest that the complexity of working memory processing may decisively contribute to the exhaustion of resources in PD patients. Increasing complexity may either affect their manipulation ability directly or impede the management of inhibitory control requirements inherent to the task.
\end{abstract}

The concept of working memory refers to the dynamic cognitive system required for the concurrent storage and processing of information (Baddeley, 1986; Cowan, 1988; Just \& Carpenter, 1992). This property is especially necessary to manage the cognitive demand that emerges when task-relevant information has to be actively maintained, monitored, and regulated in its use for guiding goal-directed behavior (Goldman-Rakic, 1987; Miyake \& Shah, 1999; Petrides, 1994). A typical example is the mental manipulation of several pieces of information to organize a sequence of actions in a flexible way as involved, for instance, in the processes of planning and problem solving. Working memory can thus be considered a fundamental component of executive control functions, which are invoked to guide behavior in situations in which habitual routines are inefficient or not even available and one has to generate novel

P. Bublak and U. Müller, Department of Neurology, Max Planck Institute of Cognitive Neuroscience, Leipzig, Germany; G. Grön, Daycare Clinic of Cognitive Neurology, University of Leipzig, Leipzig, Germany; M. Reuter, Neurological Hospital Martha-Maria Halle/Saale, Germany; D. Y. von Cramon, Department of Neurology, Max Planck Institute of Cognitive Neuroscience, and Daycare Clinic of Cognitive Neurology, University of Leipzig.

We thank Nadja Saupe for assistance in data acquisition, Anke Pitzmaus for laboratory assistance, and Torsten Schubert for valuable discussions. This work was supported by the German Bundesministerium für Bildung, Forschung und Technologie, Interdisziplinaeres Zentrum für Klinische Forschung at the University of Leipzig (Project C-9).

Correspondence concerning this article should be addressed to P. Bublak, who is now at Biological and Clinical Psychology, University of Eichstaett, Ostenstrasse 26-28, D-85072 Eichstaett, Germany. E-mail: peter.bublak@ku-eichstaett.de lines of action (Norman \& Shallice, 1986; Shallice \& Burgess, 1998; Tranel, Anderson, \& Benton, 1994).

The close connection between working memory and executive control functions is exemplified by the cognitive profile of patients suffering from Parkinson's disease (PD). It is well established that PD patients are subject to working memory deficits as revealed, for example, by delayed response tasks (Postle, Jonides, Smith, \& Corkin, 1997). Moreover, PD patients have severe difficulties especially in tasks like digit ordering that require the manipulation of information maintained in working memory (Cooper et al., 1992; Cooper, Sagar, Jordan, Harvey, \& Sullivan, 1991). These deficits may contribute substantially to the impairments observed in executive tasks like self-ordered pointing (Petrides \& Milner, 1982), spatial working memory (Morris et al., 1988; Owen, Downes, Sahakian, Polkey, \& Robbins, 1990), or planning tasks like the Tower of London (Shallice, 1982). Although these tasks have a strong strategic component and - in the case of planning tasks-require unique, single-contingency solutions, the necessity to monitor and manipulate information within working memory may underlie their special sensitivity for revealing deficits in medicated as well as untreated PD patients ${ }^{1}$ (Gabrieli, Singh, Stebbins, \& Goetz, 1996; Lange et al., 1992; Morris et al., 1988; Owen, Iddon, Hodges, Summers, \& Robbins, 1997; Owen et al., 1992). For example, Owen et al. (1992) reported longer initiation times in PD patients in the Tower of London planning task. This was interpreted as reflecting PD

\footnotetext{
${ }^{1}$ As stated by one of our reviewers, there is a complex interaction between PD and dopaminergic medications, because frontal versus striatal catecholamine depletions affect cognitive functions differently. A detailed consideration of this issue is beyond the scope of the present article, however, and the reader is referred to a recent publication by Crofts et al. (2001).
} 
patients' difficulties in preparing a sequence of moves by organizing them within working memory prior to the execution. Together with deficits in set shifting (GauntlettGilbert, Roberts, \& Brown, 1999) or the reduced ability to inhibit prepotent responses (Dujardin, Degreef, Rogelet, Defebvre, \& Destee, 1999), PD patients' impairments in tasks requiring the manipulation of information within working memory closely resemble the deficient performance of patients with frontal lobe damage suffering from a dysexecutive syndrome (Dubois \& Pillon, 1997).

From a neuroanatomical perspective, these impairments can be related to a dysfunction of the dorsolateral prefrontal cortex (DLPFC). Evidence from animal studies (Petrides, 1994, 1995) as well as from neuroimaging findings in human subjects (D'Esposito, Postle, Ballard, \& Lease, 1999; Owen, Evans, \& Petrides, 1996; Petrides, Alivisatos, Evans, \& Meyer, 1993; Petrides, Alivisatos, Meyer, \& Evans, 1993; Postle, Berger, \& D'Esposito, 1999) indicates that the DLPFC is critically involved in tasks requiring the manipulation of information maintained in working memory. The DLPFC is heavily interconnected with several anterior and posterior cortical as well as subcortical structures, spanning a widespread cerebral network to support the concurrent storage and processing of information (Carpenter, Just, \& Reichle, 2000; Fuster, 1993; Goldman-Rakic, 1987; Passingham, 1993; Petrides, 1994; Petrides \& Pandya, 1999). In $\mathrm{PD}$, the neuronal degeneration in the pars compacta of the substantia nigra (Hornykiewicz, 1966) can impair the integrity of DLPFC functions because of the disturbance within a complex dorsolateral prefrontal loop (Alexander, DeLong, \& Strick, 1986). This loop originates from the DLPFC, which is reentered via sequential projections to the dorsolateral head of the caudate nucleus, the globus pallidus, the substantia nigra, and the thalamus (Alexander et al., 1986; Chow \& Cummings, 1999; Cummings, 1993; Middleton \& Strick, 1994). A disruption of this circuit may result either from a dysfunction of the DLPFC itself, due to dopamine depletion within mesocortical projections, or from the loss of dopamine in the striatum (Dubois \& Pillon, 1997). These neuropathological mechanisms can be assumed to underlie the working memory impairment in PD that gives rise to the deficits observed in executive tasks.

The assumption that a fundamental working memory impairment is at the core of the deficient executive control functions in PD patients is further confirmed by a recent study of Gabrieli et al. (1996). These authors found PD patients' poor performance in a wide range of executive tasks to be closely related to a reduced working memory capacity, that is, a diminished amount of attentional resources to support the concurrent storage and processing of information (cf. Daneman \& Carpenter, 1980; Just \& Carpenter, 1992). Such an explanation implies that difficulties with the manipulation of working memory information arise only when the cognitive demand induced by a given task exceeds the available working memory capacity. An essential question for research, therefore, is to specify the task characteristics responsible for the exhaustion of attentional resources in PD patients. Theoretical conceptions about the constraints on working memory functioning can provide important clues in this regard.

In Baddeley's (1986) influential model, for example, which has recently been updated by Baddeley and Logie (1999), attentional limitations are ascribed to a supervisory component, the "central executive." Its role is assumed to refer to the coordination of information from subsidiary slave systems, the "phonological loop" and the "visualspatial sketchpad," devoted to the maintenance of modalityspecific information. According to this approach, which has been applied successfully to the examination of PD patients (Brown \& Marsden, 1991; Dalrymple-Alford, Kalders, Jones, \& Watson, 1994; D'Esposito \& Postle, 2000), storage and processing functions of working memory are strictly separated and maintenance of information does not contribute to the demand on attentional capacity. In contrast to this view, some authors have suggested that storage is inherent to the attention-demanding functions of working memory, casting doubt on the possibility of strictly separate maintenance and processing aspects (O'Reilly, Braver, \& Cohen, 1999). This approach is assumed to be especially important for the consideration of complex tasks involving manipulation of working memory information, for which multiple steps of processing have to be coordinated and intermediate products of computation have to be stored (Mayr \& Kliegl, 1993; O'Reilly et al., 1999; Wenger \& Carlson, 1995). It is in line with a different class of working memory models that conceptualize capacity constraints as resulting from a limited pool of resources for which both storage and processing of information compete (Anderson, Reder, \& Lebiere, 1996; Just \& Carpenter, 1992; Kimberg, D'Esposito, \& Farah, 1997; Kimberg \& Farah, 1993; Lovett, Reder, \& Lebiere, 1999).

According to the latter view, the consumption of attentional resources in tasks requiring manipulation of information within working memory will decisively be determined by the maintenance demand. That is, performance in manipulation tasks should be affected by the number of list items to be processed. In fact, it has long been known from experimental psychological studies that manipulation of working memory information declines in efficiency if the demand on item maintenance increases because of a higher number of list items to be processed (Carlson, Wenger, \& Sullivan, 1993; Weber, Burt, \& Noll, 1986). A similar relationship can also be observed in PD patients. Typically, these patients perform quite comparably to normal control participants in the self-ordered pointing and the spatial working memory tasks if only relatively few items are involved. However, in the more demanding task conditions in which item storage is more heavily strained because of larger set sizes, their deficits clearly emerge (Gabrieli et al., 1996; Lange et al., 1992; Morris et al., 1988; Owen et al., 1992, 1997).

Although a contribution of the maintenance demand to PD patients' deficits in manipulation tasks seems well established, another possibility has not hitherto been assessed sufficiently. As known from patients with frontal lobe damage, for example, deficits may result from the complexity of working memory processing required by a task, even if the 
number of items to be processed remains constant (Waltz, Knowlton, \& Holyoak, 1998; Waltz et al., 1999). Thus, the question arises of whether a similar effect will emerge in PD patients in a manipulation task. If so, PD patients will perform more poorly if manipulation is rendered more complex but the number of items remains constant. As a result, more complex processing within working memory may give rise to a longer time required for initiating a response. An answer to this question is important because it taps a typical characteristic of many executive tasks. For instance, thinking about alternative ways of solving a problem or deciding the most economic line of action frequently requires combining the same set of information in various arrangements within working memory. If this ability is restricted because of reduced attentional resources and more demanding manipulation processes are impeded, behavioral flexibility will decline and an optimal adaptation to novel situations may be constrained. The current evidence is inconclusive in this regard, however. In tasks like self-ordered pointing or spatial working memory, more demanding manipulation is confounded with a larger number of working memory items to be processed. Whereas the digit-ordering task avoids this problem, because a constant number of seven digits is used in each trial, the manipulation demand cannot be varied systematically. Applying this kind of variation was the aim of the present study.

To that end, we assessed the ability of PD patients to manipulate a constant number of items within working memory at different levels of demand. The applied paradigm was similar to the one introduced by Carlson et al. (1993), which we have already used successfully in the examination of patients suffering from severe closed-head injury (Bublak, Schubert, Matthes-von Cramon, \& von Cramon, 2000). In our task, the participants had to remember a list of four digits and use this list for guiding a sequence of forced-choice responses. The task demand was varied by the order in which the digits had to be recalled from working memory for guiding the response sequence correctly. In one condition, which was considered a baseline condition, recall of the digits was serial, and thus no reordering of the list items was necessary. In contrast, reordering of the list items was required in two further conditions, with different degrees of demand. In the first of these conditions, the digit list had to be reordered partially, and in the second condition complete reordering was required. In this way, although the demand on the manipulation of information within working memory was variable, the amount of information involved remained the same because of the constant number of list items.

This paradigm was used for examining a group of nondemented, nondepressive patients suffering from mild to moderate PD. We hypothesized that compared with a control group matched for sex, age, and education, PD patients would perform worse than normal control participants in our task conditions requiring reordering of the digits, with this deterioration being more pronounced with the increasing demand of the task. We assumed that the effects of a higher manipulation demand would be especially pronounced prior to the beginning of the response sequence, when several responses could be prepared in advance. To test the assumption that PD patients' deficits in our task are related to a reduction of working memory capacity, a reading-span task was applied. To validate our experimental paradigm with a more conventional measure, we used digit ordering as an executive task to see whether both tasks were related and whether our PD patient group performed worse than normal control participants in digit ordering as well. Digit span measures were used to exclude the possibility that our results could be explained simply by differences in short-term memory span. Finally, a simple response time task was applied to examine the role of a speed factor.

\section{Method \\ Participants}

\section{PD Participants}

Fourteen patients ( 5 men, 9 women) were selected from the Department of Neurology of the Leipzig University Hospital, Leipzig, Germany, and from the Neurological Hospital MarthaMaria Halle/Saale, Germany. Mean age was 55.1 years $(S D=$ 14.7 , range $=31-73)$. Premorbid intelligence was estimated with a German multiple-choice vocabulary test (MWT), which correlates fairly well with global IQ and is more insensitive to cerebral dysfunction than the Wechsler Adult Intelligence Scale (Lehrl, Triebig, \& Fischer, 1995). Mean IQ was estimated as 99.6 ( $S D=$ 8.9). Diagnosis of idiopathic PD was established by clinical examination of an experienced neurologist according to standard neurological criteria (Calne, Snow, \& Lee, 1992). Severity of disease was rated by means of a German translation of the full Unified Parkinson's Disease Rating Scale (UPDRS; Fahn, Elton, $\&$ Members of the UPDRS Development Committee, 1987) and a modified version of the Hoehn and Yahr scale (Hoehn \& Yahr, 1967). Mean UPDRS score was $46.9(S D=14.6$, range $=20-71)$. The median of the Hoehn and Yahr stage of PD was 2.3 (range $=1.5-3.0)$. All patients had mild or moderate symptoms of PD, with 4 patients at Stage 1.5, 3 at Stage 2, 3 at Stage 2.5, and 4 at Stage 3. Mean duration of disease onset was 47.3 months ( $S D$ $=50.0$, range $=1-156$ ). Prior to testing, patients underwent a psychiatric screening to exclude patients with depression and/or symptoms of dementia. The Hamilton Depression Rating Scale (HDRS; Hamilton, 1960) was used to verify absence of depressive symptoms. Participants with a Hamilton score above 9 would be excluded. Screening for dementia was done using the Mini Mental State Examination (MMSE; Folstein, Folstein, \& McHugh, 1975). Participants scoring below 27 would be excluded from the study. None of the patients tested showed any clinically relevant signs of depression (HDRS scores: $M=2.9, S D=2.4$, range $=0-7$ ) or dementia (MMSE scores: $M=28.9, S D=1.0$, range $=27-30$ ). Ten patients received levodopa in varying combinations with dopamine agonists (pergolide, alpha-dihydroergocryptine, cabergoline), monoamine oxidase inhibitors (selegiline), or catechol-Omethyltransferase inhibitors (entacapone or tolcapone). Levodopa administration was always in combination with a decarboxylase inhibitor. For 1 patient, medication consisted of the $N$-methyl-Daspartate antagonists amantadine and budipine, without any levodopa. Three patients were de novo cases. All medicated patients were tested within 2-4 hr after the last dose of their antiparkinsonian medication in periods without severe motor fluctuations or dyskinesias. 


\section{Normal Control Participants}

Fourteen normal control participants were tested and matched for sex ( 5 men, 9 women), age $(M=55.2, S D=14.7$, range $=$ $30-68)$, and IQ $(M=101.8, S D=8.6)$. They had no history of psychiatric or neurologic disorder or drug abuse, and there were no signs of dementia (MMSE scores: $M=29.0, S D=0.9$, range $=$ 27-30) or depression (HDRS scores: $M=1.1, S D=1.0$, range $=$ $0-3)$. Normal control participants were paid for participation.

\section{Procedure}

\section{Testing Protocol}

Participants were tested in two separate sessions. Each session lasted about $2.5 \mathrm{hr}$, including breaks, which were allowed as required by the participants. In the first session a series of neuropsychological tests was administered, not all of which are reported in this article. Tests included the revised form of the Wechsler Memory Scale (WMS-R); the Modified Card Sorting Test (Lezak, 1995); and a computerized battery of attention tasks (Testbatterie zur Aufmerksamkeitspruefung [Test Battery for the Assessment of Attention]; Zimmermann \& Fimm, 1989), including the simple response time task. The second session comprised the experimental task, assessment of working memory capacity, and the digit ordering test. Fluctuating patients were examined during on-states, and testing was interrupted when severe dyskinesias occurred.

\section{Experimental Paradigm}

Task conditions. A schematic overview of the experimental paradigm, which has been described in detail elsewhere (Bublak et al., 2000), is given in Figure 1. In each task condition, a memory list was presented during a study phase lasting $4 \mathrm{~s}$. It comprised four digits that were sequentially presented on a computer screen. The first two digits were always shown in green, and the last two digits always in red. Each digit was presented for $700 \mathrm{~ms}$, with an interstimulus interval of $300 \mathrm{~ms}$. After a short delay of $4 \mathrm{~s}$, participants had to recall the digit list to use it for guiding a sequence of forced-choice responses during the test period. The task conditions differed from each other in terms of the serial order in which the four digits had to be recalled. There were three conditions:

1. In the no-reordering condition, which was considered a baseline condition, participants had to recall the digits in the same order as presented during the study phase, that is, first green digit, second green digit, first red digit, second red digit. For example, the list $1-2-3-4$ would have to be recalled as $1-2-3-4$. (Note that in contrast to this example, which is used to give a more illustrative description of the task, ascending or descending digit lists were never presented in the experiment.)

2. In the partial-reordering condition, participants had to recall the digits in a different order from presentation. Recall was demanded in the following order: first green digit, first red digit, second green digit, second red digit. That is, the list 1-2-3-4

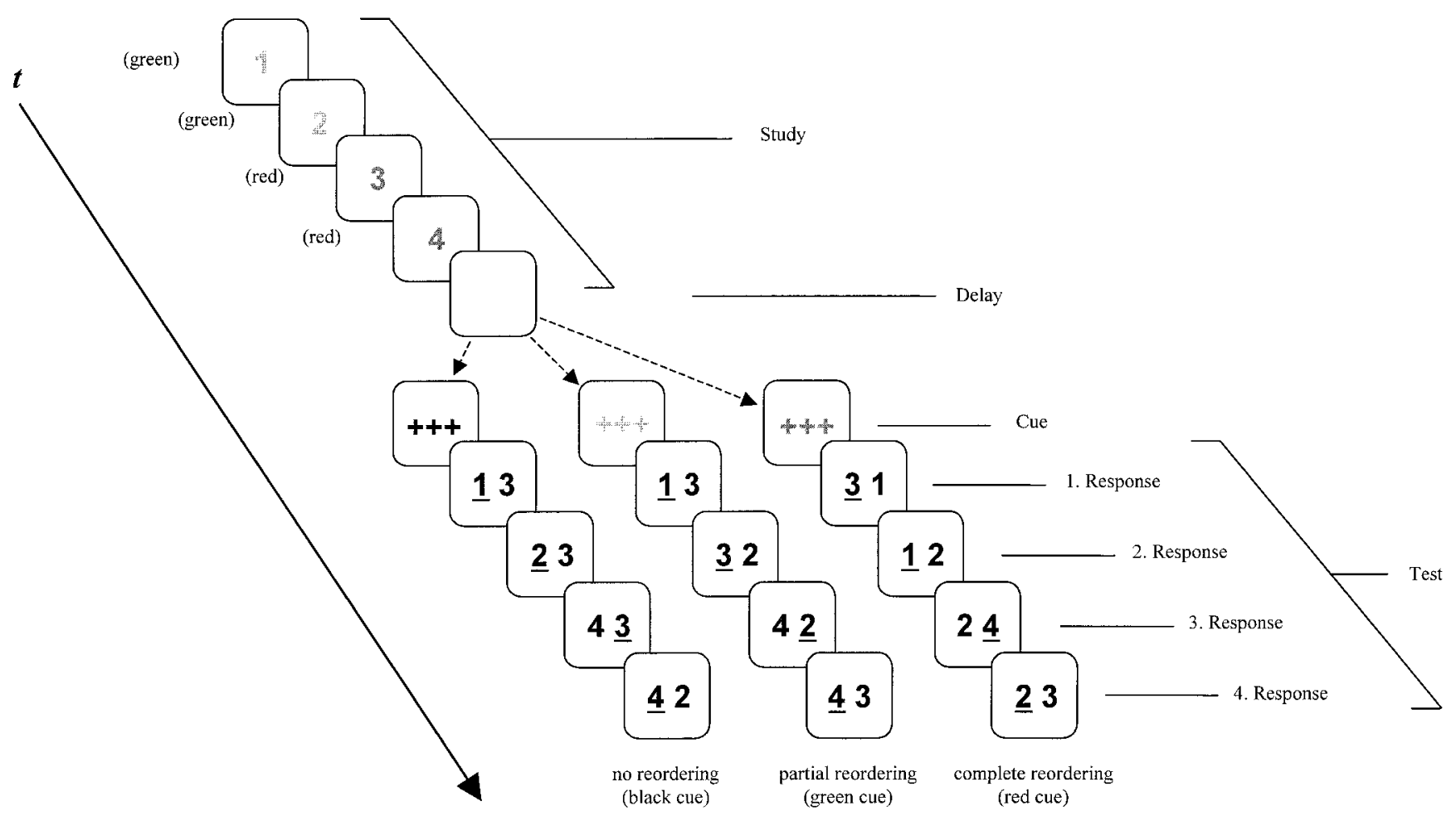

Figure 1. Schematic drawing of the manipulation task used in this study (light gray = green color; dark gray $=$ red color). Digits correct for response are underlined in the drawing, for demonstration purposes, but were not underlined in the actual experiment. (See the Method section for a detailed description of the procedure.) Note that the sequence 1-2-3-4 is only an illustrative example that was not used in the actual experiment. Instead, sequences such as $8-3-1-6$ were used. $t=$ time. 
would have to be recalled as 1-3-2-4, with the serial position of two digits altered.

3. In the complete-reordering condition, participants had to recall the digits in the following order: first red digit, first green digit, second red digit, second green digit. That is, 1-2-3-4 would have to be recalled as $3-1-4-2$, with the serial position of all four digits changed.

With each task condition, a colored cue (five horizontally arranged plus signs) was associated, which appeared after the delay period for $1 \mathrm{~s}$. Cue color was black in the no-reordering condition, green in the partial-reordering condition, and red in the completereordering condition.

Note that because of the constant coloring of digits during presentation (first two green, then two red digits), the rules to be applied for reordering in the partial-reordering and completereordering conditions were entirely complementary. Furthermore, the cue color in these two task conditions was the same as the digit color from which the correct reordering should have started according to the instruction rules.

Response sequence. During the test period of all three task conditions, participants had to execute four sequential forcedchoice responses to recall the digits from the remembered list in the order required by a given task condition. To that end, for each response a digit pair was presented on the screen, with one digit to the left and the other one to the right of center. In each pair, one digit was correct, because it matched the digit required by the order of the current task condition. The alternative digit was also a digit from the study list, but it did not match the required order and thus was incorrect for response. Participants had to select the spatial position (left or right) of the correct digit out of the pair by pressing a corresponding left or right response key. Each digit pair remained on the screen until a response was given by the participant. After the participant pressed the key, the next choice pair appeared after an interval of $100 \mathrm{~ms}$, requiring recall of the next digit. In this way, four response pairs were presented successively and participants pressed a response key four times to sequentially select the correct digits in the order required by the experimental condition. Response time latency was measured from onset of a digit pair until the participant pressed a response key. This method allowed assessment of initiation times (latency of first response) and execution times (mean interresponse times) for each trial.

The test period started after a random time interval inserted between cue presentation and appearance of the first response pair. This interval was used to prevent participants from anticipating the exact time at which the first digit pair would appear for response. It had a mean duration of $1 \mathrm{~s}$ and a maximum duration of $2 \mathrm{~s}$.

Experimental procedure. The three experimental conditions were presented as blocks with ascending difficulty. That is, the no-reordering condition was always presented first, followed by the partial-reordering and then the complete-reordering condition. This was done to preclude asymmetric transfer effects between conditions due to their different levels of difficulty. At the beginning of each experimental condition, the examiner verbally explained the task to the participants, supported by a graphical display of the task printed on a sheet of paper. Participants then performed a number of practice trials (usually 16) until they felt familiar with the task. During the practice trials, verbal feedback was given by the examiner. No feedback was provided during the actual experiment. The first eight trials of the experimental task were hidden warm-up trials, which were excluded from analysis. Each condition was divided in blocks of eight trials, after which a break was inserted, with continuation of the experiment being completely under control of the participant. Between the experimental conditions, a few minutes rest were given to participants before beginning the next condition. To minimize test duration in the experimental task for patients, some patients received only a shortened version of the experimental task. That is, for each task condition, a test duration of $20 \mathrm{~min}$ was scheduled, which is enough time for a healthy control participant to complete a condition. When 20 min were exceeded, the running block was completed until the next break occurred, and then the task condition was terminated. In this way, each patient contributed between 16 and 40 trials to each condition. Each control participant completed a full-test version, to keep experimental duration comparable to patients, but only the trials corresponding to the matched patient were used for analysis.

The experiment was conducted in a dimly lit room. The experimental session was controlled by the software package ERTS (BeriSoft Corporation, Frankfurt/Main, Germany) and run on a 586 Pentium PC, which also registered response times and errors. Participants sat in front of a computer monitor, on which the stimuli were presented. Viewing distance was approximately 80 $\mathrm{cm}$. Participants responded by pressing one of two response buttons- one left, one right - in front of them on the table. Participants were asked to press the buttons with two fingers of their preferred hand and to have these two fingers always rest upon the response buttons during the experiment. Instructions to participants stressed both speed and accuracy of response.

\section{Simple Response Time}

For the simple reaction time task, the procedure from the computerized test battery introduced by Zimmermann and Fimm (1989) was selected. Participants fixated on a small white square at the center of the screen. Each trial began with a random foreperiod of 2-5 s, after which a large white cross replaced the fixation point. Participants had to respond to the appearance of this cross as fast as possible. The response initiated the next foreperiod. Participants responded by pressing a response button with the index finger of their preferred hand, and reaction times were registered by a computer. Trials with response latencies less than $100 \mathrm{~ms}$ or more than $2 \mathrm{~s}$ were counted as invalid and repeated. There were 10 practice trials before a test session of 40 trials started.

\section{Working Memory Capacity}

Working memory capacity was assessed by a reading span task: A patient version of the test as originally introduced by Daneman and Carpenter (1980), using shorter German sentences, was applied. Two to four sentences per span length were presented without repetition. Participants read them aloud from cards with one sentence per card while being asked to remember the last word of each sentence. Correct recall of the words was rewarded by one point, independent of the order of original appearance. The range of possible scores was between two and five (see also Müller, Wächter, Barthel, Reuter, \& von Cramon, 2000).

\section{Digit Ordering Test}

The procedure introduced by Cooper et al. (1991) was applied. A string of seven digits was selected by chance (repetitions of less than three digits were allowed) and auditorily presented to the participants (e.g., 5-3-6-1-7-2-1). Participants were asked to repeat the digits, thereby reordering the string in ascending fashion (e.g., 1-1-2-3-5-6-7). There were 15 trials. For each digit placed in correct position, one point was awarded, until a response broke the ascending order or a digit could not be recalled. 


\section{Digit Span}

The conventional forward and backward versions of the Digit Span subtest from the WMS-R were administered (see, e.g., Lezak, 1995).

\section{Results \\ Experimental Task}

Accuracy and response time data were analyzed separately. For accuracy, we first compared both groups of participants (PD patients and healthy control participants) with respect to the performance in the baseline condition, using a $t$ test. Then, we assessed the impact of task demand by comparing all three task conditions within the same analysis of variance (ANOVA). Finally, to account for possible baseline differences, both manipulation conditions were compared with each other in an analysis of covariance (ANCOVA), with the baseline condition as a covariate. For response times, we first included both dependent measures (i.e., initiation time and execution time) in a multivariate analysis before performing separate ANOVAs for initiation time and execution time and the subsequent ANCOVAs. A multivariate approach was chosen to take a possible covariance between initiation time and execution time into account (cf. McDowell, Whyte, \& D'Esposito, 1997, for a similar approach).

\section{Accuracy}

Accuracy was calculated as the proportion of completely correct trials (i.e., all four responses correct) related to the total number of trials.

\section{Baseline Condition}

Mean accuracy for the no-reordering task condition was compared between groups using an unpaired $t$ test, which revealed a significant difference, $t(269)=-3.12, p<.01$. Thus, PD patients performed less accurately than normal control participants in the baseline task requiring the serial recall of digits without reordering (see Figure 2).

\section{Impact of Task Demand}

To assess the impact of task demand on performance accuracy, we conducted an ANOVA with repeated measures using task conditions (no, partial, and complete reordering) as a within-subjects and group (PD patients, normal control participants) as a between-subjects factor. The results (see Figure 2) showed a significant main effect of group, $F(1,26)=22.08, p<.01$. This effect remained significant when the ANOVA was redone as an ANCOVA, using baseline accuracy as a covariate to check for a possible confounding of the results due to baseline differences, $F(1,25)=8.70, p<.01$. The main effect of task condition, $F(2,52)=3.79, p<.05$, was also significant, whereas the Group $\times$ Task Condition interaction was not significant, $F(2,52)=0.19, p>.80$. Paired $t$ tests with Bonferroni

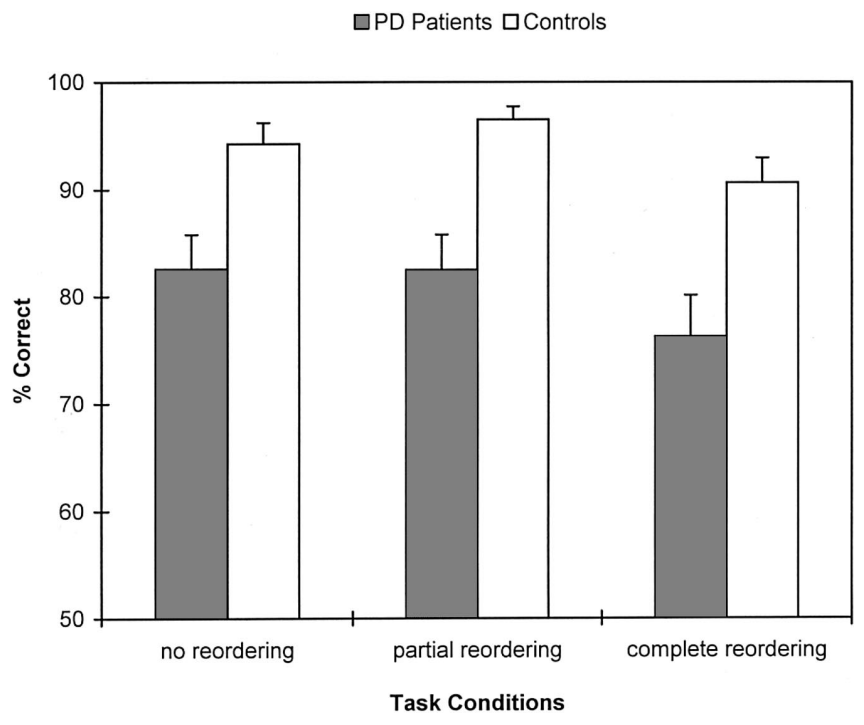

Figure 2. Mean accuracy $(+1 S E)$ as a function of task condition for Parkinson's disease (PD) patients and controls.

correction showed that across groups, performance was less accurate in the complete-reordering task condition compared with the partial-reordering condition, $t(27)=2.92$, $p<.01$, whereas the partial-reordering and no-reordering conditions did not differ significantly from each other, $t(27)=-.54, p>.50$.

Taken together, PD patients performed less accurately than normal control participants in all three task conditions, to a similar degree, and for both groups performance accuracy reliably declined in the task condition requiring complete reordering of the digits.

\section{Response Times}

Harmonic means of the correct trials were used for analysis of initiation time (latency of first response) and execution time (interresponse latencies). This was done to compensate for response time outliers without the need for data exclusion. As mentioned in the Method section, the number of observations was unequal between participants, and some participants performed only relatively few trials. Therefore, excluding extreme response times would have resulted in an overproportional loss of response times in some participants' data. ${ }^{2}$

\section{Baseline Condition}

Initiation times and execution times were compared between groups using Hotelling's multivariate $T^{2}$ test. As

\footnotetext{
${ }^{2}$ Use of harmonic means is a standard procedure to deal with reaction time outliers (see Milner, 1986). Although median reaction time would seem to be an alternative method, this procedure is considered rather problematic by some authors (e.g., J. Miller, 1988) because the distribution is not taken into account. This problem is avoided by the use of harmonic means.
} 
indicated by Figures 3 and 4, both initiation time and execution time were slower in PD patients in the no-reordering condition, and this difference between groups approached significance $\left(T^{2}=6.18\right), F(2,25)=2.97, p<$ .08 .

\section{Impact of Task Demand}

A multivariate analysis of variance with repeated measures was conducted using initiation time and execution time as the dependent measures, and group (2) and task conditions (3) as between- and within-subjects factors, respectively. There were main effects of group, Wilks's $\Lambda(1$, $26)=.72, p<.05$, and task condition, Wilks's $\Lambda(2,52)=$ $.55, p<.01$, as well as a significant Group $\times$ Task Condition interaction, Wilks's $\Lambda(2,52)=.77, p<.05$.

Separate univariate analyses for initiation and execution time were calculated, applying the Greenhouse-Geisser correction to compensate for violations of the sphericity assumption, as indicated by a significant Mauchly sphericity test (Kirk, 1995). For initiation time (see Figure 3), the ANOVA revealed significant main effects of group, $F(1$, $26)=9.31, p<.01$, and task condition, $F(2,52)=20.02$, $p<.01$, and a significant Group $\times$ Task Condition interaction, $F(2,52)=7.05, p<.01$. A rather similar pattern emerged for both groups when task conditions were compared within PD patients and normal control participants, respectively, using paired $t$ tests with Bonferroni correction. Although initiation time did not differ significantly between the no-reordering and partial-reordering conditions$t(13)=.69, p>.50$, for normal control participants, and $t(13)=1.39, p>.18$, for PD patients-there was a significant increase in initiation time between the partial-reordering and complete-reordering conditions $-t(13)=2.25, p<$

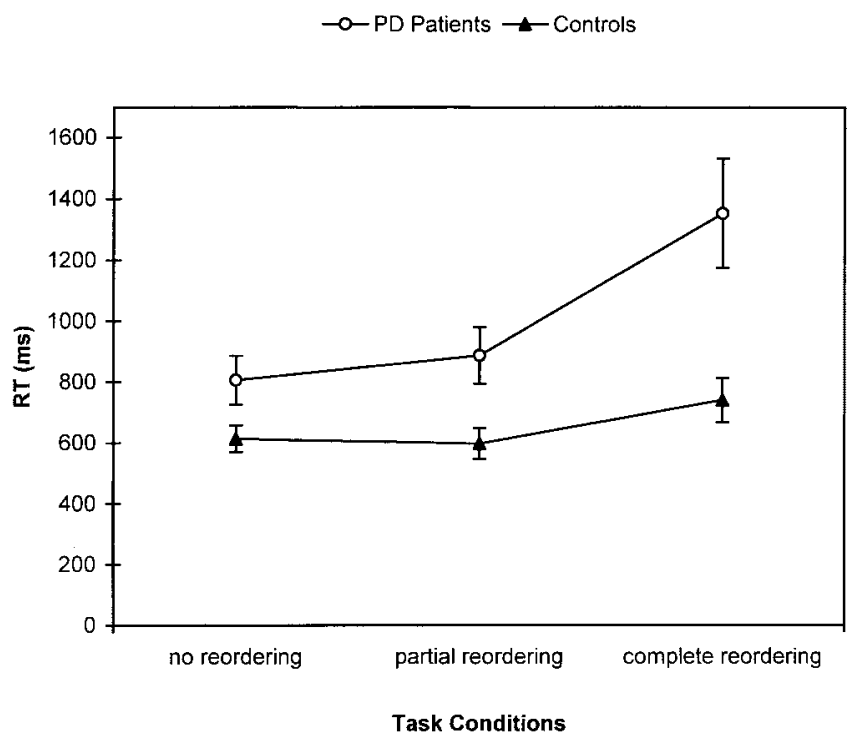

Figure 3. Mean initiation time $( \pm 1 S E)$ as a function of task condition for Parkinson's disease (PD) patients and controls. RT = reaction time.

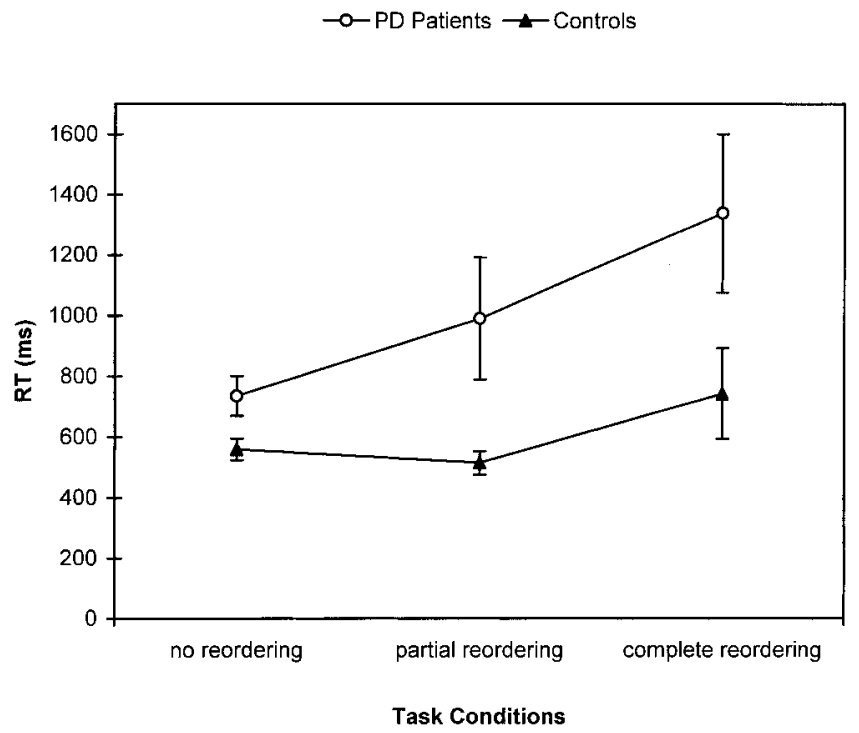

Figure 4. Mean execution time $( \pm 1 S E)$ as a function of task condition for Parkinson's disease (PD) patients and controls. RT $=$ reaction time.

.05 , for normal control participants, and $t(13)=4.77, p<$ .01 , for PD patients. This increase in initiation time was more pronounced in PD patients, however, as indicated by the significant interaction between group and task condition when only the partial-reordering and complete-reordering conditions were considered: $F(1,26)=7.75, p=.01$; group effect, $F(1,26)=9.79, p<.01$; task condition effect, $F(1$, $26)=27.31, p<.01$. To take into account a possible confounding of these results by baseline speed differences, the univariate analysis was repeated as an ANCOVA, with initiation time in the baseline condition as a covariate. The main effect of group remained significant, $F(1,25)=4.40$, $p<.05$.

For execution time (see Figure 4), the ANOVA revealed significant main effects of group, $F(1,26)=5.60, p<.05$, and task condition, $F(2,52)=7.16, p<.01$. In this case, the interaction between both factors did not reach significance, $F(2,52)=2.02, p>.15$ (power $=.55)$. Paired $t$ tests with Bonferroni correction, calculated across groups, showed that execution of the response sequence required more time in the complete-reordering condition than in the partial-reordering condition, $t(27)=-3.27, p<.01$, whereas the partial-reordering and no-reordering conditions did not differ significantly, $t(27)=-1.08, p>.25$. The slight tendency of an increasing difference between groups was not related to a more pronounced difference in the most demanding task condition, as revealed by an analysis in which only the partial-reordering and complete-reordering conditions were considered: Group $\times$ Task Condition interaction, $F(1,26)=.46, p>.50$; group effect, $F(1$, $26)=4.87, p<.05 ;$ task condition effect, $F(1,26)=10.63$, $p<.01$. Moreover, the significant main effect of group disappeared, $F(1,25)=1.06, p>.30$, when an ANCOVA was conducted using execution time in the baseline condition as a covariate to assess a speed account of the results. 
Overall, PD patients were slower at initiating and executing the response sequences in all task conditions. For both control participants and PD patients, there was no reliable increase in initiation and execution times between the no-reordering and partial-reordering task conditions, although a possible effect in PD patients for execution time may have been concealed because of low power, as suggested by Figure 4. A significant increase of initiation and execution times was found, however, between both task conditions requiring reordering of the digits, with complete reordering being more demanding than partial reordering. It is important to note that this increase was more pronounced in PD patients for initiation time, and this effect was not explainable by speed differences in the baseline task. In contrast, the increase between partial reordering and complete reordering was not stronger in PD patients for execution time, and differences between groups could be accounted for by speed differences in the baseline no-reordering task condition.

\section{Practice Effects}

As described in the Method section, some of the PD patients received a shortened version of our experimental task. Therefore, the possibility has to be considered that decreased performance of PD patients in our task conditions requiring reordering of the digits was associated with less opportunity to learn the mapping of instruction set to response set. To account for this possibility, practice effects were assessed in the partial-reordering and complete-reordering task conditions. To that end, in each of these task conditions mean initiation time, execution time, and accuracy were calculated for the first and the last block of trials for each participant. For each of these dependent measures, an ANOVA with repeated measures was conducted, with task block (first, last) as a within-subjects and group (PD, normal control participants) as a between-subjects factor. Differential practice effects between groups would be indicated by an interaction of these two factors.

For initiation time, in both the partial-reordering and the complete-reordering task conditions, there was a significant main effect of group, $F(1,26)=5.48, p<.05$, and $F(1$, 26) $=8.92, p<.01$, respectively, whereas the main effect of task block was not significant, $F(1,26)=1.03, p>.30$, and $F(1,26)=0.21, p>.60$, respectively. The Group $\times$ Task Block interaction was also not significant, $F(1,26)=$ $.44, p>.40$, and $F(1,26)=0.00, p>.95$, respectively. Thus, there was neither a reliable effect of practice on initiation time nor a differential effect of practice for PD patients or normal control participants.

A rather similar result emerged for execution time. Again, there was a significant main effect of group: partial reordering, $F(1,26)=5.59, p<.05$; complete reordering, $F(1,26)=5.49, p<.05$. The main effect of task blockpartial reordering, $F(1,26)=.30, p>.55$; complete reordering, $F(1,26)=.56, p>.45-$ and the Group $\times$ Task Block interaction-partial reordering, $F(1,26)=1.18, p>$ .25 ; complete reordering, $F(1,26)=1.89, p>.15$-were not significant. Although the nonsignificant interaction in the complete-reordering task condition might be related to our small sample size, it should be noted that, nevertheless, differences between groups with respect to execution time were explainable by speed differences in the baseline task, as shown above.

A somewhat different pattern was revealed in considering accuracy. For the complete-reordering condition, there was a significant main effect of group, $F(1,26)=5.82, p<.05$, a nonsignificant main effect of task block, $F(1,26)=1.13$, $p>.25$, and a nonsignificant Group $\times$ Task Block interaction, $F(1,26)=0.69, p>.40$. In contrast, this interaction was revealed to be significant for the partial-reordering condition: $F(1,26)=5.22, p<.05$; group effect, $F(1$, $26)=8.82, p<.01$; task block effect, $F(1,26)=0.77, p>$ .35 . Whereas normal control participants had a slight tendency to perform less accurately in the last compared with the first block of trials, $t(13)=1.17, p=.13$, PD patients improved in performance from the first to the last block, $t(13)=-1.98, p<.05$. Thus, we had to assess whether this differential practice effect could account for the more pronounced increase of initiation time for PD patients in the complete-reordering condition as compared with the partialreordering condition, as described above. Therefore, we calculated an ANCOVA on initiation time with task condition (partial and complete reordering) as a within-subjects factor, group (normal control participants, PD patients) as a between-subjects factor, and the change in accuracy from the first to the last block in the partial-reordering condition as a covariate. The main effect of group remained unaffected, $F(1,25)=7.40, p<.05$, as did the Group $\times$ Task Condition interaction: $F(1,25)=6.77, p<.05$; task condition effect, $F(1,25)=26.03, p<.01$, indicating that our results were not influenced by differential practice effects in the partial-reordering condition.

\section{Working Memory Capacity}

Within the PD group, the mean score of the working memory capacity measure was $3.13(S D=0.25)$. Within the control group, the mean score was $3.48(S D=0.40)$. As revealed by a Mann-Whitney $U$ test, this difference was significant $(U=38.5, p<.01)$. Thus, the PD group had a lower working memory capacity than the normal control group. To assess the relationship between working memory capacity and our task conditions requiring reordering of the digit list, Spearman's rank correlations were calculated, separate for each group, between the capacity measure and initiation time or execution time, respectively. To that end, initiation times and execution times were averaged across the partial-reordering and complete-reordering task conditions. Within the group of PD patients, working memory capacity and initiation time correlated significantly, although moderately $(\rho=-.55, p<.05)$. The capacity measure and execution time were similarly correlated, although this correlation did not reach significance $(\rho=$ $-.47, p<.10)$. Within the control group, no significant correlation was found, either between working memory 
capacity and initiation time $(\rho=-.08, p>.40)$ or between capacity and execution time $(\rho=-.07, p>.40)$. Results are summarized in Table 1.

\section{Digit Ordering Task}

Group comparisons with respect to the digit ordering task scores were conducted using the Mann-Whitney $U$ test. There was a significant difference between both groups $(U=45.5, p<.01)$. PD patients achieved a lower score $(M=65.4, S D=12.0)$ than control participants $(M=76.5$, $S D=12.7)$. To assess the relationship between the digit ordering task and our experimental tasks requiring reordering of the digits, we again computed Spearman's rank correlations. As before, initiation times and execution times were collapsed across the partial-reordering and completereordering task conditions. Within the patient group, digit ordering scores were significantly correlated with initiation time $(\rho=-.57, p<.05)$ but not with execution time $(\rho=$ $-.07, p>.40)$. The reverse pattern was found within the control group (initiation time: $\rho=-.30, p>.15$; execution time: $\rho=-.55, p<.05)$. Results are summarized in Table 1.

\section{Digit Span}

The raw scores of Digit Span forward and backward, respectively, were analyzed. Mean Digit Span forward was $6.6(S D=0.9)$ for the PD patient group and 6.4 $(S D=1.3)$ for the control group. A Mann-Whitney $U$ test confirmed that this difference was not significant $(U=92.5$, $p>.35)$. For Digit Span backward, PD patients tended to score below control participants $(U=69.0, p<.09)$, achieving a mean score of $4.3(S D=0.6)$ compared with $4.9(S D=1.2)$ for normal control participants. Results are summarized in Table 1.

\section{Simple Reaction Time}

Mean response times in the simple reaction time task were analyzed with an unpaired $t$ test. There was a significant difference between both groups, $t(26)=2.25, p<.05$. $\mathrm{PD}$ patients responded more slowly $(M=329.2, S D=$
122.0) than control participants $(M=250.6, S D=47.2)$. Simple response time was not significantly correlated with working memory capacity either within the PD patient group $(\rho=-.33, p>.10)$ or within the control group $(\rho=$ $.17, p>.30)$. No significant correlations were found between simple response time and execution time in the reordering conditions (averaged across the partial-reordering and complete-reordering conditions) in PD patients $(r=$ $.29, p>.15)$ or in normal control participants $(r=.17, p>$ .30). Within the PD patient group, however, simple response time was significantly correlated with initiation time ( $r=.61, p<.05)$, a result not found in control participants $(r=.32, p>.15)$. Results are summarized in Table 1 .

\section{Performance of De Novo Patients}

The three de novo cases were very comparable to medicated patients with respect to age (de novo: $M=54.33$, $S D=17.79$; medicated: $M=55.27, S D=14.70)$ and IQ (de novo: $M=99.50, S D=9.46$; medicated: $M=100.17$, $S D=7.86)$. Except for initiation time in the no-reordering baseline condition and for execution time in the completereordering task condition, mean performance of de novo cases was superior to that of medicated patients for all variables tested. In most cases the differences were not striking, with the greatest difference arising for the digit ordering task (de novo: $M=71.00, S D=8.72$; medicated: $M=63.91, S D=12.64$ ) and Digit Span backward (de novo: $M=4.67, S D=0.29$; medicated: $M=4.18$, $S D=0.64)$. However, mean initiation times in the partialreordering experimental task conditions (de novo: $M=$ $670.00 \mathrm{~ms}, S D=320.43$; medicated: $M=945.55$, $S D=$ 342.97 ) and the complete reordering condition (de novo: $M$ $=1,118.00, S D=328.25$; medicated: $M=1,416.55, S D=$ 732.68) were substantially faster for de novo patients, as was mean execution time in the partial-reordering condition (de novo: $M=687.00, S D=399.33$; medicated: $M$ $=1,072.09, S D=818.57)$. A clear-cut difference emerged for accuracy in the no-reordering baseline condition (de novo: $M=96.90, S D=3.10$; medicated: $M=78.70$, $S D=10.37)$. Here, all three de novo cases performed above the 75th percentile of the medicated patients' performance.

Table 1

Mean Values and Correlations for the Different Tasks Assessed in Parkinson's Disease (PD) Patients and Controls

\begin{tabular}{|c|c|c|c|c|c|c|c|c|}
\hline \multirow[b]{2}{*}{ Task } & \multicolumn{2}{|c|}{$M$ values $(S D \mathrm{~s})$} & \multicolumn{3}{|c|}{ Correlations within PD group } & \multicolumn{3}{|c|}{ Correlations within control group } \\
\hline & PD patients & $\begin{array}{l}\text { Normal } \\
\text { controls }\end{array}$ & $\begin{array}{l}\text { Simple } \\
\text { reaction } \\
\text { time }\end{array}$ & $\begin{array}{l}\text { Initiation } \\
\text { time } \\
\text { (Reord.) }\end{array}$ & $\begin{array}{c}\text { Execution } \\
\text { time } \\
\text { (Reord.) }\end{array}$ & $\begin{array}{c}\text { Simple } \\
\text { reaction } \\
\text { time }\end{array}$ & $\begin{array}{l}\text { Initiation } \\
\text { time } \\
\text { (Reord.) }\end{array}$ & $\begin{array}{l}\text { Execution } \\
\text { time } \\
\text { (Reord.) }\end{array}$ \\
\hline WM capacity & $3.13 * *(0.25)$ & $3.48(0.40)$ & -.33 & $-.55^{*}$ & $-.47 \dagger$ & .17 & -.08 & -.07 \\
\hline Digit ordering & $65.4 * *(12.0)$ & $76.5(12.7)$ & & $-.57 *$ & -.07 & & -.30 & $-.55^{*}$ \\
\hline Digit Span forward & $6.6(0.9)$ & $6.4(1.3)$ & & & & & & \\
\hline Digit Span backward & $4.3 \dagger(0.6)$ & $4.9(1.2)$ & & & & & & \\
\hline $\begin{array}{l}\text { Simple reaction time } \\
(\mathrm{ms})\end{array}$ & $329.2 *(122.0)$ & $250.6(47.2)$ & & $.61 *$ & .29 & & .32 & .17 \\
\hline
\end{tabular}

Note. $\quad$ Reord. $=$ combined score for the partial-reordering and complete-reordering task conditions; $\mathrm{WM}=$ working memory.

$\dagger p<.10$ (marginally significant). $* p<.05$. ** $p<.01$. 


\section{Discussion}

In the present study, we were interested in answering the question of whether the performance of PD patients in a task requiring manipulation of a list of working memory elements would be affected by the task demand irrespective of the number of these elements. The experimental paradigm applied required participants to remember a list of four digits and to use it for guiding a sequence of forced-choice responses by transposing either none, half, or all of the digits in the list with respect to their original serial order. In accordance with our hypothesis, task performance of PD patients declined overproportionally with the increasing task demand, compared with a matched group of healthy control participants. More precisely, the increase of initiation time from the partial-reordering condition to the complete-reordering condition was more pronounced in PD patients than in normal control participants. In agreement with the assumption that PD patients' difficulties in our task are related to a reduction of working memory capacity, reading span scores were lower for our patient group compared with normal control participants, and the scores significantly correlated with our experimental task conditions requiring manipulation. Moreover, these conditions were related to the results of the digit ordering task, in which PD patients also performed worse than normal control participants, indicating that manipulation ability was validly tapped by our experimental task. A comparable performance between PD patients and normal control participants was revealed for the Digit Span forward task, excluding the possibility of explaining our findings by group differences in short-term memory span. Finally, the relevance of a speed factor is suggested by PD patients' slower responses in a simple reaction time task, which was associated with initiation times but not with working memory capacity in our experimental manipulation conditions in PD patients.

These results confirm previous findings that PD patients are impaired in tasks requiring manipulation of information within working memory, whereas their short-term memory functions remain largely unaffected (Cooper et al., 1991, 1992; Lange et al., 1992; Morris et al., 1988; Owen et al., 1992, 1997). However, our results can also extend these earlier findings because of the experimental variation induced by the applied paradigm. In contrast to other studies assessing manipulation of working memory information in PD patients, we were able to selectively vary the manipulation requirement in our task conditions without changing the number of list items to be processed. Hence, our results can complement previous findings by specifying that besides the number of items involved, the degree to which a serial reordering of working memory elements is necessary represents a decisive determinant for the demand on attentional working memory resources. It is suggested that the differential task demand induced by our experimental paradigm increasingly strained attentional working memory resources, giving rise to an overproportional increase of initiation time in PD patients because of their reduced working memory capacity.
It could be argued that the manipulation demand is not varied independently from the maintenance demand in our task. Whereas the number of list items (i.e., digits) to be remembered remained identical, the three task conditions might differ with respect to the additional demand to maintain the color associated with each digit, the instruction set (i.e., the manipulation rule), and the response set. Such an account certainly applies when both task conditions requiring reordering of the digits are compared with the noreordering baseline condition. However, it seems less plausible that, likewise, the partial-reordering and completereordering conditions differed from each other in this regard, for the following reasons. First, in both these conditions, coloring of the digits was identical in each trial, because the first two digits were always green and the last two digits always red. Therefore, the association to be maintained between a digit's color and its identity was the same within both conditions. Second, the manipulation rules in both conditions were completely complementary to each other. That is, partial reordering required starting with the first green digit and then recalling the digits by alternating between colors, and the only difference in complete reordering was that recall had to begin with the first red digit. Hence, we would suggest that the instruction set in the partial-reordering condition (i.e., "first green digit, first red digit, second green digit, second red digit") involved a similar maintenance demand as the instruction set in the complete-reordering condition (i.e., "first red digit, first green digit, second red digit, second green digit"). Finally, with respect to the response sets, we would claim that both conditions were comparable because four responses had to be given in either case, guided by the four digits from the list. A difference in response sets would have occurred only if each digit from the list had been associated with a specific finger and, thus, a particular finger sequence had to be executed depending on the digit list. However, this was not the case in the present study. Taken together, we would claim that in the partial-reordering and complete-reordering task conditions the manipulation demand was varied successfully by the number of digits to be transposed with respect to their original serial order, whereas the demand on maintenance of information was kept comparable between both conditions as far as possible in our experimental paradigm.

Nevertheless, a higher maintenance demand might still have resulted from differential practice effects. That is, if PD patients compared with normal control participants had less opportunity to acquire a manipulation rule efficiently, especially in the complete-reordering task condition, the demand to keep the rule active in working memory would not be comparable between groups. This would lead to stronger effects of task demand in PD patients in the most demanding task condition. This possibility is particularly important in the face of the evidence that PD patients are less able than healthy participants to gain an advantage from practice, for example, while learning a problem-solving procedure (Saint-Cyr, Taylor, \& Lang, 1988). Moreover, some of our PD patients received only a shortened version of our experimental task, possibly impeding the efficient 
acquisition of the manipulation rules. Our analysis of practice effects runs against such an assumption, however. If anything, PD patients had slightly more difficulties in acquiring the rule in the easier manipulation condition, as indicated by the improvement of accuracy from the first to the last block of trials within the partial-reordering condition but not in the more demanding complete-reordering condition. This result would run against our finding of a more pronounced increase of the effects of task demand from the less to the more demanding task condition in PD patients. In fact, our analysis of the effects of task demand on initiation time revealed that the differences between groups remained significant when differential practice effects in the partial-reordering case were considered. Therefore, although some difficulties in acquiring the manipulation rules might have been present in PD patients, they cannot account for our finding of a differential impact of task demand on patients' initiation times.

Our findings lend further support to the assumption put forward by Gabrieli et al. (1996) that cognitive deficits in PD patients are related to a reduction of working memory capacity. These authors further hypothesized that this capacity reduction can partly be ascribed to a slowing of processing speed. This assumption seems to be not well supported by our results, because performance in the simple response time task, which was used as a speed measure, was not significantly correlated with the reading span reflecting working memory capacity. It should be noted, however, that a simple response time procedure itself may not necessarily provide an appropriate index of response speed. As suggested by the work of Goodrich, Henderson, and Kennard (1989), for instance, simple response time deficits in PD patients could reflect the impairment of an attention-demanding, strategic control process governing the perceptuomotor system. Frith and Done (1986) ascribed this process to the necessity of focusing attention to maintain a readiness to make a particular response. Similarly, a response in our experimental task conditions required participants to focus attention to maintain a readiness to respond to a particular stimulus (i.e., the target digit). This was especially necessary for the first response (initiation time), for which the appearance of the imperative stimulus was temporally unpredictable, just as in the simple response time task. As a result, the significant correlation between the simple response time task and initiation time in our manipulation conditions appears plausible. Moreover, it seems less surprising to find a low correlation between simple response time and the reading span task, which did not involve the strategic process just described.

The purpose of the present study was to vary a manipulation task with respect to the complexity of working memory processing, inducing differential demands on attentional resources. The significant, albeit moderate, correlation between working memory capacity and our experimental manipulation conditions in PD patients suggests that this variation was successful. However, it still leaves open the question of the nature of the association between working memory capacity and the complexity of the manipulation task. A first possibility is that a capacity reduction impedes complex manipulation processes directly. Following Mayr and Kliegl (1993, p. 1298), our task variation can be considered to tap different levels of coordinative complexity induced by the amount to which parallel mental activities for maintenance and processing of information have to be coordinated to regulate and monitor the information flow between interrelated processing steps. According to this explanation, our results would imply that coordinative complexity, and consequently the amount of attentional resources required, increases with the number of working memory items that have to be transposed with respect to their original serial order. This is in line with the assumption of Waltz et al. (1999, p. 124) that complexity results from the necessity to integrate relations within working memory, for which the relative temporal order of multiple stimuli is a critical example. When a complete reordering of working memory elements is required, as was the case in our most complex task condition, a capacity reduction may raise problems like those claimed by Mayr and Kliegl (1993), that is, loss of critical information during processing and redundant processing due to the reiteration of unsuccessful processing steps. These authors proposed such an account to explain the cognitive decline in the elderly, and a similar explanation may, in addition to the slowing of processing steps, be applicable to the problems found in PD patients in our study.

There is evidence to suggest that the selection of an item within working memory may be one major cause for the demand on attentional resources. For example, Dark (1990) and Garavan (1998) have demonstrated that in normal participants, substantial processing costs are invoked by the requirement to select a particular item within working memory from among several represented elements. Following E. K. Miller (1999), Rowe, Toni, Josephs, Frackowiack, and Passingham (2000) have recently proposed that such "attentional selection" of an item within working memory is a core mechanism underlying executive functions, which is closely related to dorsolateral prefrontal activity. Our results suggest that attentional selection can be graded in its demand on attentional resources. That is, selection seems to be increasingly demanding as more items have to be selected in a different order than originally presented and, consequently, re-presented within working memory. Put in different terms, the degree of incongruence between the "input order" and the "output order" of working memory elements may determine the demand on working memory capacity.

A second possible explanation for the correlation between working memory capacity and manipulation complexity is that increasing complexity exhausts attentional resources in PD patients, giving rise to an inefficient management of other control processes that are independent from manipulation itself. In this regard, it is worth noting that our task involved some aspects that are different from those immanent to tasks like digit ordering. More specifically, our task included a forced-choice response component that places demands on, for example, stimulus discrimination and response selection. Moreover, we used repeated stimuli in our working memory lists and distracting items in our response pairs. Both aspects are known to put a strong 
demand on interference-resolving processes (Fuster, 1985, 1990). As proposed by Pennington, Bennetto, McAleer, and Roberts (1996) and Engle, Kane, and Tuholski (1999), for example, a decline of working memory capacity can give rise to a weakening of inhibitory mechanisms required to suppress irrelevant information or prepotent responses. It is possible, therefore, that the response mode used in our experimental task was an important feature to reveal PD patients' difficulties. That is, to the degree that attentional resources were exhausted because of the complexity of working memory processing, PD patients' ability to manage inhibitory control demands included in our task may have been significantly reduced. Both explanations would be compatible with the assumption that an increasing complexity of the manipulation of working memory information overproportionally strains PD patients' attentional resources and can decisively contribute to their executive deficits.

\section{References}

Alexander, G. E., DeLong, M. R., \& Strick, P. L. (1986). Parallel organization of functionally segregated circuits linking basal ganglia and cortex. Annual Review of Neuroscience, 9, 357-381.

Anderson, J. R., Reder, L. M., \& Lebiere, C. (1996). Working memory: Activation limitations on retrieval. Cognitive Psychology, 30, 221-256.

Baddeley, A. (1986). Working memory. New York: Oxford University Press.

Baddeley, A. D., \& Logie, R. H. (1999). Working memory: The multiple-component model. In A. Miyake \& P. Shah (Eds.), Models of working memory: Mechanisms of active maintenance and executive control (pp. 28-61). Cambridge, England: Cambridge University Press.

Brown, R. G., \& Marsden, C. D. (1991). Dual task performance and processing resources in normal participants and patients with Parkinson's disease. Brain, 114, 215-231.

Bublak, P., Schubert, T., Matthes-von Cramon, G., \& von Cramon, D. Y. (2000). Differential demands on working memory for guiding a simple action sequence: Evidence from closed-head injured subjects. Journal of Clinical and Experimental Neuropsychology, 22, 176-190.

Calne, D. B., Snow, B. J., \& Lee, C. (1992). Criteria for diagnosing Parkinson's disease. Annals of Neurology, 32, 125-127.

Carlson, R. A., Wenger, J. L., \& Sullivan, M. A. (1993). Coordinating information from perception and working memory. Journal of Experimental Psychology: Human Perception and Performance, 19, 531-548.

Carpenter, P. A., Just, M. A., \& Reichle, E. D. (2000). Working memory and executive function: Evidence from neuroimaging. Current Opinion in Neurobiology, 10, 195-199.

Chow, T. W., \& Cummings, J. L. (1999). Frontal-subcortical circuits. In B. L. Miller \& J. L. Cummings (Eds.), The human frontal lobes (pp. 3-26). New York: Guilford Press.

Cooper, J. A., Sagar, H. J., Doherty, S. M., Jordan, N., Tidswell, P., \& Sullivan, E. V. (1992). Different effects of dopaminergic and anticholinergic therapies on cognitive and motor function in Parkinson's disease. Brain, 115, 1701-1725.

Cooper, J. A., Sagar, H. J., Jordan, N., Harvey, N. S., \& Sullivan, E. V. (1991). Cognitive impairment in early, untreated Parkinson's disease and its relationship to motor disability. Brain, 114, 2095-2122.
Cowan, N. (1988). Evolving conceptions of memory storage, selective attention, and their mutual constraints within the human information-processing system. Psychological Bulletin, 104, 163-191.

Crofts, H. S., Dalley, J. W., Collins, P., Van Denderen, J. C. M., Everitt, B. J., Robbins, T. W., \& Roberts, A. C. (2001). Differential effects of 6-OHDA lesions of the frontal cortex and caudate nucleus on the ability to acquire an attentional set. Cerebral Cortex, 11, 1015-1026.

Cummings, J. L. (1993). Frontal-subcortical circuits and human behavior. Archives of Neurology, 50, 873-880.

Dalrymple-Alford, J. C., Kalders, A. S., Jones, R. D., \& Watson, R. W. (1994). A central executive deficit in patients with Parkinson's disease. Journal of Neurology, Neurosurgery, and Psychiatry, 57, 360-367.

Daneman, M., \& Carpenter, P. A. (1980). Individual differences in working memory and reading. Journal of Verbal Learning and Verbal Behavior, 19, 450-466.

Dark, V. J. (1990). Switching between memory and perception: Moving attention or memory retrieval? Memory \& Cognition, 18, 119-127.

D'Esposito, M., \& Postle, B. R. (2000). Neural correlates of processes contributing to working-memory function: Evidence from neuropsychological and pharmacological studies. In S. Monsell (Ed.), Attention and performance: XVIII. Control of cognitive processes (pp. 579-602). Cambridge, MA: MIT Press.

D’Esposito, M., Postle, B. R., Ballard, D., \& Lease, J. (1999). Maintenance versus manipulation of information held in working memory: An event-related fMRI study. Brain and Cognition, 41, 66-86.

Dubois, B., \& Pillon, B. (1997). Cognitive deficits in Parkinson's disease. Journal of Neurology, 244, 2-8.

Dujardin, K., Degreef, J. F., Rogelet, P., Defebvre, L., \& Destee, A. (1999). Impairment of the supervisory attentional system in early untreated patients with Parkinson's disease. Journal of Neurology, 246, 783-788.

Engle, R. W., Kane, M. J., \& Tuholski, S. W. (1999). Individual differences in working memory capacity and what they tell us about controlled attention, general fluid intelligence, and functions of the prefrontal cortex. In A. Miyake \& P. Shah (Eds.), Models of working memory: Mechanisms of active maintenance and executive control (pp. 102-134). Cambridge, England: Cambridge University Press.

Fahn, S., Elton, R. L., \& Members of the UPDRS Development Committee. (1987). Unified Parkinson's Disease Rating Scale. In S. Fahn, C. D. Marsden, D. Caine, \& M. Goldstein (Eds.), Recent developments in Parkinson's disease (Vol. 2, pp. 153163). Florham Park, NJ: Macmillan Healthcare Information.

Folstein, M. F., Folstein, S. E., \& McHugh, P. R. (1975). Minimental state: A practical method for grading the cognitive status of patients for the clinician. Journal of Psychiatry Research, 12, 189-198.

Frith, C. D., \& Done, D. J. (1986). Routes to action in reaction time tasks. Psychological Research, 48, 169-177.

Fuster, J. M. (1985). The prefrontal cortex, mediator of crosstemporal contingencies. Human Neurobiology, 4, 169-179.

Fuster, J. M. (1990). Prefrontal cortex and the bridging of temporal gaps in the perception-action cycle. In A. Diamond (Ed.), Annals of the New York Academy of Sciences: Vol. 608. The development and neural bases of higher cognitive functions (pp. 318-336). New York: New York Academy of Sciences.

Fuster, J. M. (1993). Memory cells in primate cortex and the activation of memory networks. In T. Ono, L. R. Squire, M. E. 
Raichle, D. I. Perrett, \& M. Fukuda (Eds.), Brain mechanisms of perception and memory: From neuron to behavior (pp. 426444). New York: Oxford University Press.

Gabrieli, J. D. E., Singh, J., Stebbins, G. T., \& Goetz, C. G. (1996). Reduced working memory span in Parkinson's disease: Evidence for the role of a frontostriatal system in working and strategic memory. Neuropsychology, 10, 322-332.

Garavan, H. (1998). Serial attention within working memory. Memory \& Cognition, 26, 263-276.

Gauntlett-Gilbert, J., Roberts, R. C., \& Brown, V. J. (1999). Mechanisms underlying attentional set-shifting in Parkinson's disease. Neuropsychologia, 37, 605-616.

Goldman-Rakic, P. S. (1987). Circuitry of primate prefrontal cortex and regulation of behavior by representational memory. In F. Plum, V. B. Mountcastle, \& S. R. Geiger (Eds.), Handbook of physiology: Section I. The nervous system: Volume V. Higher functions of the brain (Part 1, pp. 373-417). Bethesda, MD: American Physiological Society.

Goodrich, S., Henderson, L., \& Kennard, C. (1989). On the existence of an attention-demanding process peculiar to simple reaction time: Converging evidence from Parkinson's disease. Cognitive Neuropsychology, 6, 309-331.

Hamilton, M. (1960). A rating scale for depression. Journal of Neurology, Neurosurgery, and Psychiatry, 23, 56-62.

Hoehn, M. M., \& Yahr, M. D. (1967). Parkinsonism: Onset, progression, and mortality. Neurology, 17, 427-442.

Hornykiewicz, O. (1966). Dopamine (3-hydroxytyramine) and brain function. Pharmacological Reviews, 18, 925-964.

Just, M. A., \& Carpenter, P. A. (1992). A capacity theory of comprehension: Individual differences in working memory. Psychological Review, 99, 122-149.

Kimberg, D. Y., D’Esposito, M., \& Farah, M. J. (1997). Cognitive functions in the prefrontal cortex-Working memory and executive control. Current Directions in Psychological Science, 6, $185-192$.

Kimberg, D. Y., \& Farah, M. J. (1993). A unified account of cognitive impairments following frontal lobe damage: The role of working memory in complex, organized behavior. Journal of Experimental Psychology: General, 122, 411-428.

Kirk, R. E. (1995). Experimental design: Procedures for the behavioral sciences. Pacific Grove, CA: Brooks/Cole.

Lange, K. W., Robbins, T. W., Marsden, C. D., James, M., Owen, A. M., \& Paul, G. M. (1992). L-Dopa withdrawal in Parkinson's disease selectively impairs cognitive performance in tests sensitive to frontal lobe dysfunction. Psychopharmacology, 107, 394-404.

Lehrl, S., Triebig, G., \& Fischer, B. (1995). Multiple choice vocabulary test MWT as a valid and short test to estimate premorbid intelligence. Acta Neurologica Scandinavica, 91, 335-345.

Lezak, M. (1995). Neuropsychological assessment. New York: Oxford University Press.

Lovett, M. C., Reder, L. M., \& Lebiere, C. (1999). Modeling working memory in a unified architecture: An ACT-R perspective. In A. Miyake \& P. Shah (Eds.), Models of working memory: Mechanisms of active maintenance and executive control (pp. 135-182). Cambridge, England: Cambridge University Press.

Mayr, U., \& Kliegl, R. (1993). Sequential and coordinative complexity: Age-based processing limitations in figural transformations. Journal of Experimental Psychology: Learning, Memory, and Cognition, 19, 1297-1320.
McDowell, S., Whyte, J., \& D'Esposito, M. (1997). Working memory impairments in traumatic brain injury: Evidence from a dual-task paradigm. Neuropsychologia, 35, 1341-1353.

Middleton, F. A., \& Strick, P. L. (October 21, 1994). Anatomical evidence for cerebellar and basal ganglia involvement in higher cognitive function. Science, 266, 458-461.

Miller, E. K. (1999). The prefrontal cortex: Complex neural properties for complex behavior. Neuron, 22, 15-17.

Miller, J. (1988). A warning about median reaction time. Journal of Experimental Psychology: Human Perception and Performance, 14, 539-543.

Milner, A. D. (1986). Chronometric analysis in neuropsychology. Neuropsychologia, 24, 115-128.

Miyake, A., \& Shah, P. (1999). Toward unified theories of working memory: Emerging general consensus, unresolved theoretical issues, and future research directions. In A. Miyake \& P. Shah (Eds.), Models of working memory: Mechanisms of active maintenance and executive control (pp. 442-481). Cambridge, England: Cambridge University Press.

Morris, R. G., Downes, J. J., Sahakian, B. J., Evenden, J. L., Heald, A., \& Robbins, T. W. (1988). Planning and spatial working memory in Parkinson's disease. Journal of Neurology, Neurosurgery, and Psychiatry, 51, 757-766.

Müller, U., Wächter, T., Barthel, H., Reuter, M., \& von Cramon, D. Y. (2000). Striatal $\left[{ }^{123} \mathrm{I}\right] \beta$-CIT SPECT and prefrontal cognitive functions in Parkinson's disease. Journal of Neural Transmission, 107, 303-319.

Norman, D. A., \& Shallice, T. (1986). Attention to action: Willed and automatic control of behavior. In R. J. Davidson, G. E. Schwartz, \& D. Shapiro (Eds.), Consciousness and self-regulation: Advances in research and theory (Vol. 4, pp. 1-18). New York: Plenum Press.

O'Reilly, R. C., Braver, T. S., \& Cohen, J. D. (1999). A biologically based computational model of working memory. In A. Miyake \& P. Shah (Eds.), Models of working memory: Mechanisms of active maintenance and executive control (pp. 375411). Cambridge, England: Cambridge University Press.

Owen, A. M., Downes, J. J., Sahakian, B. J., Polkey, C. E., \& Robbins, T. W. (1990). Planning and spatial working memory following frontal lobe lesions in man. Neuropsychologia, 28, 1021-1034.

Owen, A. M., Evans, A. C., \& Petrides, M. (1996). Evidence for a two-stage model of spatial working memory processing within the lateral frontal cortex: A positron emission tomography study. Cerebral Cortex, 6, 31-38.

Owen, A. M., Iddon, J. L., Hodges, J. R., Summers, B. A., \& Robbins, T. W. (1997). Spatial and non-spatial working memory at different stages of Parkinson's disease. Neuropsychologia, 35, $519-532$.

Owen, A. M., James, M., Leigh, P. N., Summers, B. A., Marsden, C. D., Quinn, N. P., et al. (1992). Fronto-striatal cognitive deficits at different stages of Parkinson's disease. Brain, 115, 1727-1751.

Passingham, R. (1993). The frontal lobes and voluntary action. Oxford, England: Oxford University Press.

Pennington, B. F., Bennetto, L., McAleer, O.. \& Roberts, R. J., Jr. (1996). Executive functions and working memory: Theoretical and measurement issues. In G. R. Lyon \& N. A. Krasnegor (Eds.), Attention, memory, and executive function (pp. 327348). Baltimore: Brookes Publishing.

Petrides, M. (1994). Frontal lobes and working memory: Evidence from investigations of the effects of cortical excisions in nonhuman primates. In F. Boller \& J. Grafman (Eds.), Handbook of neuropsychology (Vol. 9, pp. 59-82). Amsterdam: Elsevier. 
Petrides, M. (1995). Impairments on nonspatial self-ordered and externally ordered working memory tasks after lesions of the mid-dorsal part of the lateral frontal cortex in the monkey. Journal of Neuroscience, 15, 359-375.

Petrides, M., Alivisatos, B., Evans, A. C., \& Meyer, E. (1993). Dissociation of human mid-dorsolateral from posterior dorsolateral frontal cortex in memory processing. Proceedings of the National Academy of Sciences, USA, 90, 873-877.

Petrides, M., Alivisatos, B., Meyer, E., \& Evans, A. C. (1993). Functional activation of the human frontal cortex during the performance of verbal working memory tasks. Proceedings of the National Academy of Sciences, USA, 90, 878-882.

Petrides, M., \& Milner, B. (1982). Deficits on subject-ordered tasks after frontal and temporal-lobe lesions in man. Neuropsychologia, 20, 249-262.

Petrides, M., \& Pandya, D. N. (1999). Dorsolateral prefrontal cortex: Comparative cytoarchitectonic analysis in the human and the macaque brain and corticocortical connection patterns. European Journal of Neuroscience, 11, 1011-1036.

Postle, B. R., Berger, J. S., \& D’Esposito, M. (1999). Functional neuroanatomical double dissociation of mnemonic and executive control processes contributing to working memory performance. Proceedings of the National Academy of Sciences, USA, 96, 12959-12964.

Postle, B. R., Jonides, J., Smith, E. E., \& Corkin, S. (1997). Spatial, but not object, delayed response is impaired in early Parkinson's disease. Neuropsychology, 11, 171-179.

Rowe, J., Toni, I., Josephs, O., Frackowiack, R., \& Passingham, R. (June 2, 2000). The prefrontal cortex: Response selection or maintenance within working memory? Science, 288, 15561560.

Saint-Cyr, J. A., Taylor, A. E., \& Lang, A. E. (1988). Procedural learning and neostriatal dysfunction in man. Brain, 111, 941959.
Shallice, T. (1982). Specific impairments of planning. Philosophical Transactions of the Royal Society of London B, 298, 199209.

Shallice, T., \& Burgess, P. (1998). The domain of supervisory processes and the temporal organization of behaviour. In A. C. Roberts, T. W. Robbins, \& L. Weiskrantz (Eds.), The prefrontal cortex: Executive and cognitive functions (pp. 22-35). New York: Oxford University Press.

Tranel, D., Anderson, S. W., \& Benton, A. (1994). Development of the concept of "executive function" and its relationship to the frontal lobes. In F. Boller \& J. Grafman (Eds.), Handbook of neuropsychology (Vol. 9, pp. 125-148). Amsterdam: Elsevier.

Waltz, J. A., Knowlton, B. J., \& Holyoak, K. J. (1998). Relational complexity, the central executive, and prefrontal cortex. Behavioral and Brain Sciences, 21, 846-847.

Waltz, J. A., Knowlton, B. J., Holyoak, K. J., Boone, K. B., Mishkin, F. S., de Menezes Santoa, M., et al. (1999). A system for relational reasoning in human prefrontal cortex. Psychological Science, 10, 119-125.

Weber, R. J., Burt, D. B., \& Noll, N. C. (1986). Attention switching between perception and memory. Memory \& Cognition, 14, $238-245$.

Wenger, J. L., \& Carlson, R. A. (1995). Learning and the coordination of sequential information. Journal of Experimental Psychology: Human Perception and Performance, 21, 170-182.

Zimmermann, P., \& Fimm, B. (1989). Neuropsychologische Testbatterie zur Erfassung von Aufmerksamkeitsdefiziten [Neuropsychological Test Battery for the Assessment of Attention Deficits]. Freiburg, Germany: Psychologisches Institut der Universität Freiburg.

Received September 13, 2001

Revision received November 30, 2001

Accepted May 6, 2002 